\title{
Comparison of linkage analysis methods for genome-wide scanning of extended pedigrees, with application to the TG/HDL-C ratio in the Framingham Heart Study
}

\author{
Benjamin D Horne*1,2, Alka Malhotra ${ }^{3}$ and Nicola J Camp ${ }^{1}$
}

Address: ${ }^{1}$ Genetic Epidemiology, Department of Medical Informatics, University of Utah School of Medicine, Salt Lake City, Utah, USA, ${ }^{2}$ Cardiovascular Department, LDS Hospital, Intermountain Health Care, Salt Lake City, Utah, USA and ${ }^{3}$ Department of Human Genetics, University of Utah School of Medicine, Salt Lake City, Utah, USA

Email: Benjamin D Horne* - benjamin@genepi.med.utah.edu; Alka Malhotra - amalhotr@genetics.utah.edu; Nicola J Camp - nicki@genepi.med.utah.edu

* Corresponding author

from Genetic Analysis Workshop 13: Analysis of Longitudinal Family Data for Complex Diseases and Related Risk Factors New Orleans Marriott Hotel, New Orleans, LA, USA, November I I-14, 2002

Published: 31 December 2003

BMC Genetics 2003, 4(Suppl I):S93

This article is available from: http://www.biomedcentral.com/I47|-2I56/4/s|/S93

\begin{abstract}
Background: High triglycerides (TG) and low high-density lipoprotein cholesterol (HDL-C) jointly increase coronary disease risk. We performed linkage analysis for TG/HDL-C ratio in the Framingham Heart Study data as a quantitative trait, using methods implemented in LINKAGE, GENEHUNTER (GH), MCLINK, and SOLAR. Results were compared to each other and to those from a previous evaluation using SOLAR for TG/HDL-C ratio on this sample. We also investigated linked pedigrees in each region using by-pedigree analysis.

Results: Fourteen regions with at least suggestive linkage evidence were identified, including some that may increase and some that may decrease coronary risk. Ten of the 14 regions were identified by more than one analysis, and several of these regions were not previously detected. The best regions identified for each method were on chromosomes 2 (LOD = 2.29, MCLINK), 5 (LOD = 2.65, GH), 7 (LOD = 2.67, SOLAR), and 22 (LOD = 3.37, LINKAGE). By-pedigree multi-point LOD values in MCLINK showed linked pedigrees for all five regions, ranging from 3 linked pedigrees (chromosome 5) to 14 linked pedigrees (chromosome 7), and suggested localizations of between $9 \mathrm{cM}$ and $27 \mathrm{cM}$ in size.

Conclusion: Reasonable concordance was found across analysis methods. No single method identified all regions, either by full sample LOD or with by-pedigree analysis. Concordance across methods appeared better at the pedigree level, with many regions showing by-pedigree support in MCLINK when no evidence was observed in the full sample. Thus, investigating by-pedigree linkage evidence may provide a useful tool for evaluating linkage regions.
\end{abstract}

\section{Background}

Obesity, diabetes, and hypertension are closely associated with low levels of high-density lipoprotein cholesterol (HDL-C) and elevated levels of triglycerides (TG), and are recognized as jointly increasing coronary risk [1]. These factors are the major components of the metabolic syndrome as outlined in the report of the National Cholesterol Education Program's Adult Treatment Panel (ATP) III [2]. 
In an evaluation of a genetic component of the metabolic syndrome, Shearman et al. [3] reported a genome-wide scan for loci linked to TG/HDL-C ratio using Framingham Heart Study (FHS) family data and SOLAR [4], which performs variance-components analysis. SOLAR allows for pedigrees of arbitrary size by estimating multi-point identity by descent (IBD) probabilities from the exact twopoint IBDs, which are then used in variance components linkage statistic calculations. SOLAR assumes multivariate normality, but is considered model-free and relies on little prior knowledge of the underlying genetic model.

In contrast, parametric linkage analysis requires specification of the underlying model of genetic inheritance. These models are usually unknown and must be estimated. Commingling analysis can be used to estimate genetic parameters from the phenotypic data. Although parametric analysis requires model specification, it can provide statistical power beyond that of model-free analyses [5]. Three linkage software packages able to perform parametric analyses include LINKAGE [6], GENEHUNTER [7], and MCLINK [8].

LINKAGE calculates exact two-point IBD probabilities for use in two-point linkage analysis, and can be used for pedigrees of arbitrary size. Two-point analysis is less sensitive to misspecification of the model parameters than multipoint, since these are absorbed into the maximization over the recombination fraction, $\theta$. For a detailed description see Göring and Terwilliger [9]. Two-point analysis, however, can be sensitive to false positives, due to misspecification of marker allele frequencies or rare alleles segregating in some families, or have low power, due to poor IBD probability estimates.

Parametric analysis in GENEHUNTER (GH) also is an exact likelihood method. Multi-point LOD analysis that is less sensitive to inaccurate allele frequencies and is superior at determining IBD probabilities can be calculated; however, multi-point LOD statistics are constrained for $\theta$ $=0$ and thus can be prone to false-negative results (loss of power) if the model parameters are misspecified. Further, the pedigree size capacity for GH is limited and with large pedigrees can require extensive trimming, which can lead to loss in power due to the elimination of important genealogical and segregational information.

MCLINK is a Markov chain Monte Carlo (MCMC) method that uses blocked Gibbs sampling to estimate multi-point IBD probabilities on extended pedigree structures. In addition, MCLINK supports a robust multi-point theta-LOD (TLOD) statistic $[9,10]$. The TLOD is a hybridmulti-point statistic that uses multi-point IBD probabilities estimated from all available marker data, but calculates the LOD statistic under a two-point paradigm, thus combining the benefits of the two-point analysis without losing haplotype information.

Thus, while multi-point analysis methods have become popular, exact multi-point methods cannot evaluate large, extended pedigrees as exact two-point methods can. Estimation methods may circumvent these issues, but may have their own weaknesses. The major objective of this study was to compare the linkage analysis results of LINKAGE, GH, and SOLAR to a potentially more robust parametric method, MCLINK, that incorporates all available pedigree information into the linkage analysis.

\section{Methods \\ Phenotype}

The data for this study consisted of real FHS data for both the original and offspring cohorts, as provided in Problem 1 of the Genetic Analysis Workshop 13 (GAW13) data set. As noted by Shearman et al. [3], cholesterol measurements were made on 12-14 h fasting blood samples. TG concentrations were measured only once for FHS original cohort participants, at Exams 10, 11, or 12. HDL cholesterol measurements from the same exam as these TG levels were used to compute the TG/HDL-C ratio. For the offspring cohort, TG and HDL-C were measured at each of the first five study cycles. The TG/HDL-C ratio was calculated for each of these cycles and the lowest ratio was selected as the one to be used in the study. The rationale was that the lowest value would generally represent measurements that were less encumbered by environmental factors. Because cholesterol values tend to become more extreme with age, this process resulted in essentially all TG/HDL-C values being drawn from offspring study cycle 1 or 2 ; thus the time frame of the cholesterol measurements was the early 1970 s for both original and offspring cohorts. The TG/HDL-C ratio was normalized using the natural logarithm to reduce skewing of the distribution.

Prior to linkage analysis, the TG/HDL-C ratio was adjusted by regression for age, body mass index (BMI), systolic blood pressure (SBP), and glucose level because of the definition of the metabolic syndrome in the ATP III guidelines [2], with BMI serving as an obesity-related surrogate for waist circumference. The same or nearest exam for which these measures were available was used in the analyses. The regression residuals were taken to represent the TG/HDL-C ratio free from the effects of the other factors (correlation of TG/HDL-C and these residuals was $r=$ 0.80 ). Adjustment for smoking and alcohol was not used because they are not components of the metabolic syndrome and they had essentially no effect on the TG/HDL$\mathrm{C}$ ratio (correlation of residuals before and after adjustment for smoking and alcohol was very high, $r=0.996$ ). Physical activity and estrogen replacement therapy were not available in the GAW13 FHS data set and thus could 
not be controlled as before [3]. Further, lipid-lowering medication use was not available in the GAW13 data set. Out of the 2885 FHS participants in the 330 available pedigrees of the genome scan, 2467 participants had an evaluable TG/HDL-C ratio and 2,461 had measured values for all factors.

\section{Genetic model}

We used commingling analysis to determine genetic parameters assuming normal distributions under a major gene model. Commingling analysis revealed significant evidence for two populations with mean TG/HDL-C residuals of 1.26 and -0.08 , with common standard deviation 0.56 . Gene frequencies of 0.01 and 0.1 were used for the dominant and recessive models. Four parametric models (HighDom, HighRec, LowDom, and LowRec) were analyzed as we were interested in locating susceptibility genes for both low (protective) and high (risk) TG/HDL-C ratios. All LOD and TLOD scores were maximized over the proportion of families linked using heterogeneity LODs $(\alpha)$.

\section{By-pedigree support}

We used by-pedigree classical multi-point LOD statistics (with $\theta=0$ ) available from MCLINK to assess if a pedigree was linked to a region. Each region was defined as the 20 cM surrounding the peak full sample LOD. A pedigree was considered linked to that region if a pedigree LOD $>0.588$ was observed, which corresponds to a nominal $p$-value of 0.05 . The region size was estimated using the 1 -LOD drop from the peak of the summed pedigree LODs (sumLOD) for linked pedigrees only, and also from the observed recombinants. We used the pedigree multi-point graphs to estimate positions of recombinants, and used a drop of 0.5 LOD units to indicate the existence of a recombinant.

\section{Results}

\section{Full sample linkage analyses}

General characteristics of the study population are given in Table 1. Overall, 14 regions were found to have at least suggestive linkage with LOD scores above 1.9 [11] by at least one analysis (Table 2).

Table I: Population characteristics for the GAWI3 FHS Cohorts.

\begin{tabular}{|c|c|c|c|}
\hline Characteristic & Overall & Original & Offspring \\
\hline Age (years) & $46.0 \pm 14.8$ & $59.8 \pm 7.4$ & $39.4 \pm 12.7$ \\
\hline Sex (male) & $48.5 \%$ & $46.7 \%$ & $49.4 \%$ \\
\hline TG/HDL-C ratio & $1.8 \pm 2.0$ & $3.0 \pm 2.7$ & $1.3 \pm 1.2$ \\
\hline Systolic blood pressure (mm Hg) & $126 \pm 20$ & $|38 \pm 2|$ & $120 \pm 16$ \\
\hline Body mass index $\left(\mathrm{kg} / \mathrm{m}^{2}\right)$ & $26 \pm 4$ & $27 \pm 4$ & $25 \pm 4$ \\
\hline Glucose $(\mathrm{mg} / \mathrm{dL})$ & $98 \pm 22$ & $98 \pm 30$ & $98 \pm 16$ \\
\hline
\end{tabular}

Table 2: Regions with at least suggestive evidence under one analysis. $A$

\begin{tabular}{|c|c|c|c|c|c|c|}
\hline Chr & cM & Nearest Marker & LINKAGE & GH & MCLINKB & SOLARC \\
\hline 1 & 276 & $203 y g 9$ & $-D$ & - & 1.93 (276 cM, HD) & - \\
\hline 2 & $20 \mathrm{I}-05$ & GATA65C03 & $1.26(205 \mathrm{cM}, \mathrm{Ir})$ & - & $2.29(20 \mathrm{l} \mathrm{cM}, \mathrm{Ir})$ & - \\
\hline 3 & $|35-4|$ & GATA84BI2 & - & - & 1.74 (135 cM, HD) & $\begin{array}{l}1.89(\mathrm{~m} ; 141 \mathrm{cM}) \\
2.50(2 ; 135 \mathrm{cM})\end{array}$ \\
\hline 3 & 194 & GATA3H0I & 1.95 (195 cM, Hr) & - & 1.98 (194 cM, Hr) & - \\
\hline 5 & 103 & GATA69HI2 & - & 2.34 (I03 cM, HD) & I.5I (103 cM, HD) & - \\
\hline 5 & 125 & ATA4DI0 & 1.45 (I25 cM, HD) & 2.65 (I25 cM, HD) & 1.57 (I 25 cM, HD) & - \\
\hline 7 & $48-57$ & GATAI3GII & - & 1.97 (52 cM, HD) & - & - \\
\hline 7 & $|6|-69$ & GATA32CI2 & - & $\mathrm{I} .52(\mathrm{I} 6 \mathrm{I} \mathrm{cM}, \mathrm{Hr})$ & I.I7 (I56 cM, HD) & $\begin{array}{l}2.12(\mathrm{~m} ; 169 \mathrm{cM}) \\
2.67(2 ; 163 \mathrm{cM})\end{array}$ \\
\hline 8 & 176 & UT72I & - & $2.26(\mathrm{I} 76 \mathrm{cM}, \mathrm{Ir})$ & - & - \\
\hline 10 & $32-33$ & ATA3IGII & $1.82(32 \mathrm{cM}, \mathrm{ID})$ & - & $1.94(33$ cM, Ir) & - \\
\hline 13 & 36 & GATA86H0I & $2.12(36 \mathrm{cM}, \mathrm{ID})$ & - & - & - \\
\hline 17 & $126-29$ & $044 \times g 3$ & $1.12(128 \mathrm{cM}, \mathrm{Ir})$ & $2.10(129 \mathrm{cM}, \mathrm{Ir})$ & $1.44(\mathrm{I} 26 \mathrm{cM}, \mathrm{Ir})$ & $1.13(2 ; 127 \mathrm{cM})$ \\
\hline 19 & $0-11$ & GATA44FIO & $1.63($ I I cM, ID) & $2.07(0 \mathrm{cM}, \mathrm{ID})$ & 1.61 (0 cM, ID) & - \\
\hline 22 & 21 & $\operatorname{Mfd} 3 \mid 3$ & 3.37 (2I cM, Ir) & - & - & $1.28(2 ; 21 \mathrm{cM})$ \\
\hline
\end{tabular}

AA region is reported only when at least one analysis produced a score over 1.9. In those regions all other analyses that reached at least a score $>$ I.0 are also reported. BH, loci linked to high TG/HDL ratio; I, low TG/HDL-C ratio; D, dominant model; $r$, recessive model. CBoth two- and multipoint variance component analyses were performed in SOLAR ( 2 indicates two-point, $m$ indicates multipoint results). DIndicates no score above I.0. 
Table 3: Regions with at least suggestive evidence under one analysis. ${ }^{A}$

\begin{tabular}{lcccc}
\hline & LINKAGE & GH & MCLINK & SOLAR \\
Chromosome & 22 & 5 & 2 & 7 \\
Full Sample LOD & $3.37(2 \mathrm{pt})$ & $2.65(\mathrm{mpt})$ & $2.29(\mathrm{mpt})$ & $2.67(2 \mathrm{pt})$ \\
$\mathrm{cM}$ & $21 \mathrm{cM}$ & $125 \mathrm{cM}$ & $201 \mathrm{cM}$ & $163 \mathrm{cM}$ \\
No. peds linked & 9 & 3 & 11 & 5 \\
sumLOD & $6.28(\mathrm{mpt})$ & $3.16(\mathrm{mpt})$ & $7.23(\mathrm{mpt})$ & $3.01(\mathrm{mpt})$ \\
I-LOD size & $13 \mathrm{cM}$ & $15 \mathrm{cM}$ & $21 \mathrm{cM}$ & $15 \mathrm{cM}$ \\
I-LOD boundaries & $12-25 \mathrm{cM}$ & $117-132 \mathrm{cM}$ & $192-213 \mathrm{cM}$ & $162-177 \mathrm{cM}$ \\
Recomb size & $27 \mathrm{cM}$ & $13 \mathrm{cM}$ & $9 \mathrm{cM}$ \\
Recomb boundaries & $0-27 \mathrm{cM}$ & $114-127 \mathrm{cM}$ & $201-219 \mathrm{cM}$ & $161-170 \mathrm{cM}$ \\
No. peds (L,R bnds) & $(0,3)$ & $(1,1)$ & $(2,3)$ & $(I, I)$
\end{tabular}

For the parametric analyses, regions were identified under both the high and low TG/HDL-C ratio models. Analyses evaluating potential loci predisposing to a high TG/HDL$\mathrm{C}$ ratio identified five potential regions on chromosomes $1,3,5$, and 7 (Table 2). The highest scores (2.34 and 2.65) were on chromosome 5 at $103 \mathrm{cM}$ and $125 \mathrm{cM}$, both identified using GH with a dominant model. The locus at 125 cM was located by all three parametric methods, the second, at $103 \mathrm{cM}$, was also located using MCLINK.

Parametric analyses to locate predisposition loci for low TG/HDL-C ratio, which could indicate a genetically protective effect, identified seven regions on chromosomes 2 , $8,10,13,17,19$, and 22 (Table 2). The best two-point score from LINKAGE was 3.37 on chromosome 22 (21 $\mathrm{cM}$, recessive model). This locus was not located by other parametric methods, but was identified by SOLAR twopoint analysis. The highest multi-point score was 2.29 on chromosome 2 (210-17 cM, recessive model) and was identified by MCLINK. This locus was also located by LINKAGE (1.26 at $205 \mathrm{cM})$.

Model-free variance components analysis identified two further regions on chromosomes 3 and 7 (Table 2). These regions were both also located by MCLINK, and GH located the region on chromosome 7 at approximately $160 \mathrm{cM}$.

\section{By-pedigree linkage evidence}

We followed up the best regions for each analysis method on chromosomes 2 (201 cM, MCLINK), $5(125 \mathrm{cM}, \mathrm{GH})$, 7 (163 cM, SOLAR), and 22 (21 cM, LINKAGE) using multi-point by-pedigree support as provided by MCLINK. Despite using only MCLINK to identify linked pedigrees, at least three linked pedigrees (LOD > 0.588) were found for each region. For each of the regions analyzed, no conflicting recombinants were evident. Table 3 illustrates the characteristics of the regions, including the number of linked pedigrees found for each region, the peak sumLOD (sum of the multi-point LODs for the linked pedigrees) and the region size and boundaries both as estimated by the 1-LOD drop and from inspection of estimated recombinants; in addition, the number of pedigrees that define the left and right recombinant boundaries are given. The smallest region (9 cM) was on chromosome 7, using recombinant boundaries, however only one recombinant on each side defined this region. The best recombinant evidence was for the region on chromosome 2 where five recombinants (two on the left hand side and three on the right) defined an 18-cM region. In addition, this region obtained the highest sumLOD (7.23), although this defined the largest 1-LOD drop $(21 \mathrm{cM})$.

\section{Conclusions}

Several loci were found by multiple analysis methods, including a region on chromosome 17 identified by all methods. This is reassuring, although a large range of LODs was often seen for the same locus. Across all 14 regions identified, only four loci were identified using only a single analysis method (one LINKAGE, two GH, and one MCLINK), six regions were identified with two methods, three regions were identified with three methods, and one region on chromosome 17 at $126-129 \mathrm{cM}$ was located by all four methods, although only GH identified the region with at least suggestive evidence. This region on chromosome 17 was also the best region observed by Klos et al. [12] for a related total cholesterol/ HDL ratio phenotype (LOD: 2.48), although Klos et al. did not have any other TG- or HDL-C-related phenotypes with LOD > 1.9.

In the analyses here, no single method identified all the regions. MCLINK performed the best in this regard with LODs > 1.0 in 10 of the total 14 regions, and 3 of the 4 regions missed were those identified by only a single method.

Shearman et al. [3], using SOLAR to analyze a related TG/ HDL-C ratio phenotype, reported one region with suggestive evidence for linkage on chromosome 7 at $155 \mathrm{cM}$ 
$(\mathrm{LOD}=2.5)$, and a further four regions with $\mathrm{LOD}>1.0$ on chromosomes 3 (LOD 1.8 at $140 \mathrm{cM}$ ), 11 (LOD 1.1 at 125 cM), 16 (LOD 1.1 at $75 \mathrm{cM}$ ), and 20 (LOD 1.7 at $35 \mathrm{cM}$ ). Our SOLAR results similarly identified regions on chromosomes 3 and 7 with comparable magnitude (Table 2).

In general, better agreement was found in this study between the parametric methods than with the results from SOLAR, and in particular between LINKAGE and MCLINK. This may be due to the similarity in pedigree structures analyzed. In addition, MCLINK agreed better with GH than it did LINKAGE. The better agreement of GH with MCLINK may be due to the multi-point nature of both analyses. Further, MCLINK obtained better agreement with SOLAR than the other two parametric methods. However, our model-free SOLAR results were more concordant with those of Shearman et al. [3] than with the parametric findings.

Even if a much less stringent LOD threshold were imposed on the analyses of these data, no single method used in this study would have identified all 14 regions here. MCLINK identified only moderate-size peaks compared across parametric methods, but located evidence for linkage (LOD > 1.0) for 10 of the total 14 regions, and had the best concordance with each of the other methods, LINKAGE located 8 out of 14 regions, GH 7 out of the total 14, and SOLAR only 4 . Of the 7 locations it found, $\mathrm{GH}$ found them all with LOD > 1.5. The lower number located by GH may be partly due to loss of pedigree structure.

The best four regions identified by each method were followed up with by-pedigree analysis using MCLINK multipoint LODs. Despite the lack of highly confirmatory full sample scores, the by-pedigree support identified at each locus was good (at least three pedigrees linked to each region). This may indicate better consistency of LOD findings at the pedigree level.

Other evaluations of linkage for TG and HDL-C phenotypes have been published, in addition to those of Shearman et al. [3] and Klos et al. [12] that are mentioned above, although only Shearman et al. have addressed the ratio of TG/HDL-C. This study replicated a finding by Peacock et al. [13] for HDL-C on chromosome 13 at $27.54 \mathrm{cM}$ (LOD: 2.36 ), although it did not replicate their other suggestive finding on chromosome 5 (39.89 cM, LOD: 3.64). Pajunkanta et al. [14] reported a region on chromosome 2 at $186 \mathrm{cM}$ for TG among Finnish families, but their other region on chromosome 10 was not replicated by this study. Among that Finnish population, Soro et al. [15] later reported on HDL-C, but none of their regions coincided with the current study's findings. A study of both
HDL-C and TG as separate measures by Coon et al. [16] also had no coincident findings.

The generally poor concordance between this study's findings and the results of studies other than Shearman et al. [3] may be due to the use of the ratio of TG and HDL-C, which is regarded as a marker of insulin resistance or the metabolic syndrome, and not just elevated cholesterol. The current study evaluated a larger population with more families than most other studies, though, and also examined loci for low TG/HDL-C ratio as a potentially protective factor, whereas other studies have only addressed loci linked to increased risk lipids (elevated TG or reduced HDL-C).

This study has identified loci linked to TG/HDL-C ratio that may relate to the metabolic syndrome or insulin resistance, including some that may increase coronary risk and some that decrease risk. Further, it indicates that multi-point and two-point analyses, based on exact inheritance probabilities (LINKAGE, GH) and estimation methods (SOLAR, MCLINK) IBD, and parametric (LINKAGE, GH, MCLINK) and model-free (SOLAR) analyses can all contribute to the identification of loci for complex quantitative traits. Although we attempted no formal comparison using simulations that would enable definitive conclusions about the comparison of methods, these results do provide a valuable practical comparison of approaches. We found reasonable consistency across multiple methods, and suggest that consistency of findings, in addition to LOD peak size, could be used in identifying potential regions. Our results indicate that the MCMC method, MCLINK, behaves as one might expect for a method that has parallels with both two-point full pedigree analyses and limited pedigree structure multi-point analyses, with reasonable correspondence with both methods. Our results further suggest the utility of inspecting by-pedigree support for regions, both to increase confidence and to increase region definition and narrowing.

\section{References}

I. Ballantyne CM, Olsson AG, Cook TJ, Mercuri MF, Pedersen TR, Kjekshus J: Influence of low high-density lipoprotein cholesterol and elevated triglyceride on coronary heart disease events and response to simvastatin therapy in 4S. Circulation 200I, 104:3046-305I.

2. Expert Panel on Detection Evaluation and Treatment of High Blood Cholesterol in Adults: Executive summary of the third report of the National Cholesterol Education Program (NCEP) Expert Panel on Detection, Evaluation, and Treatment of High Blood Cholesterol in Adults (Adult Treatment Panel III). JAMA 200I, 285:2486-2497.

3. Shearman AM, Ordovas JM, Cupples LA, Schaefer EJ, Harmon MD, Shao Y, Keen JD, DeStefano AL, Joost O, Wilson PWF, Housman DE, Myers $\mathrm{RH}$ : Evidence for a gene influencing the TG/HDL-C ratio on chromosome 7q32,3-qter: a genome-wide scan in the Framingham Study. Hum Molec Genet 2000, 9:1315-1320.

4. Almasy L, Blangero J: Multipoint quantitative-trait linkage analysis in general pedigrees. Am J Hum Genet 1998, 62:1198-1211. 
5. Greenberg DA, Abreu PC: Determining trait locus position from multipoint analysis: accuracy and power of three different statistics. Genet Epidemiol 200I, 21:299-3।4.

6. Lathrop GM, Lalouel JM, White RL: Construction of human linkage maps: likelihood calculations for multipoint analysis. Genet Epidemiol 1986, 3:39-52.

7. Kruglyak L, Daly MJ, Reeve-Daly MP, Lander ES: Parametric and nonparametric linkage analysis: a unified multipoint approach. Am J Hum Genet 1996, 58: I347-I 363.

8. Thomas A, Gutin A, Abkevich V, Bansal A: Multipoint linkage analysis by blocked Gibbs sampling. Stat Comput 2000, 10:259-269.

9. Göring $\mathrm{HH}$, Terwilliger JD: Linkage analysis in the presence of errors. I: Complex-valued recombination fractions and complex phenotypes. Am J Hum Genet 2000, 66: 1095-1 106.

10. Abkevich V, Camp NJ, Gutin A, Farnham JM, Cannon-Albright L, Thomas A: Using a robust multipoint linkage statistic (TLOD) to map complex trait loci. Genet Epidemiol 200I, 2 I:492-497.

II. Lander E, Kruglyak L: Genetic dissection of complex traits: guidelines for interpreting and reporting linkage results. Nat Genet 1995, I I:24I-247.

12. Klos KL, Kardia SL, Ferrell RE, Turner ST, Boerwinkle E, Sing CF: Genome-wide linkage analysis reveals evidence of multiple regions that influence variation in plasma lipid and apolipoprotein levels associated with risk of coronary heart disease. Arterioscler Thromb Vasc Biol 2001, 21:971-978.

13. Peacock JM, Arnett DK, Atwood LD, Myers RH, Coon H, Rich SS, Province MA, Heiss G, on behalf of the Investigators of the NHLBI Family Heart Study: Genome scan for quantitative trait loci linked to high-density lipoprotein cholesterol. The NHLBI Family Heart Study. Arterioscler Thromb Vasc Biol 200I, 2I:1823-1828

14. Pajunkanta P, Terwilliger JD, Perola M, Hiekkalinna T, Nuotio I, Ellonen P, Parkkonen M, Hartiala J, Ylitalo K, Pihlajamaki J, Porkka K, Laakso M, Viikari J, Ehnholm C, Taskinen MR, Peltonen L: Genomewide scan for familial combined hyperlipidemia genes in Finnish families, suggesting multiple susceptibility loci influencing triglyceride, cholesterol, and apolipoprotein B levels. Am J Hum Genet 1999, 64: I453-I463.

15. Soro A, Pajukanta P, Lilja HE, Ylitalo K, Hiekkalinna T, Perola M, Cantor RM, Viikari JSA, Taskinen MR, Peltonen L: Genome scans provide evidence for low-HDL-C loci on chromosomes 8q23, 16q24. I-24.2, and 20q I 3.I I in Finnish families. Am J Hum Genet 2002, 70:1333-1340.

16. Coon H, Leppert MF, Eckfeldt JH, Oberman A, Myers RH, Peacock JM, Province MA, Hopkins PN, Heiss G: Genome-wide linkage analysis of lipids in the hypertension genetic Epidemiology Network (HyperGEN) blood pressure study. Arterioscler Thromb Vasc Biol 200I, 21:1969-1976.

Publish with Bio Med Central and every scientist can read your work free of charge

"BioMed Central will be the most significant development for disseminating the results of biomedical research in our lifetime. "

Sir Paul Nurse, Cancer Research UK

Your research papers will be:

- available free of charge to the entire biomedical community

- peer reviewed and published immediately upon acceptance

- cited in PubMed and archived on PubMed Central

- yours - you keep the copyright
BioMedcentral 